\section{Malignant Renal Epithelioid Angiomyolipoma with Liver Metastases Managed with Transarterial Chemoembolization (TACE): A Rare Case Report and Review}

\begin{abstract}
Renal epithelioid angiomyolipoma (EAML) is a rare tumor with the potential for malignant metastasis. EAML with local invasion is radiologically difficult to differentiate from renal cell carcinoma (RCC). The treatment of choice is surgery and there is no known effective management dealing with distant metastases. This case report described the imaging features of EAML and assessed the efficacy of TACE for the management of liver metastases, which, to our knowledge, no previous literature had addressed this issue.
\end{abstract}

Keywords: Malignant epithelioid angiomyolipoma; Perivascular epithelioid cell tumor; Liver metastasis; Transarterial chemoembolization

Received: July 09, 2018; Accepted: August 06, 2018; Published: August 13, 2018

\section{Introduction}

Renal angiomyolipoma ( $A M L$ ) has been recognized as a mesenchymal tumor and contains variable portion of smooth muscle cells, dysmorphic vessels, and adipose tissues. As defined by the World Health Organization in 2004, it belongs to the perivascular epithelioid cell tumor (PEComa) family shared by the distinctive morphology of smooth muscle cells which are epithelioid and cluster around the vessels [1-3]. It is a rare mesenchymal tumor belonging to the PEComa family and is associated with the tuberous sclerosis complex with two recognized forms: classic renal $A M L$ (CAML) being more common with a more benign course, and its rarer and more malignant counterpart EAML. CAML is the most common tumor of the kidney which accounts for approximately $2-6.4 \%$ of the resected renal tumors with benign behavior [4]. CAML shows variable size with typical gross or macroscopic fatty component. EAML is described as a rare variant of AML and presented histologically with abundant epithelioid cells and malignant potential, and, radiologically, with little or no fatty component, relatively larger tumor size, solid part with hyperattenuation on pre-contrast computed tomography (CT) and rapid wash-in and slow wash-out on dynamic phase, and more aggressive features like RCC. Based on pathological features, EAML can't be well differentiated from CAML and shares the same picture of immunohistological stain, with positive myogenic [smooth muscle actin (SMA)] stain. But,
Zih-Cen Lin', I-li Lin', ChuKuang Chou ${ }^{2}$, Cheng-Huang Shen ${ }^{3}$, Wen-Chuang Wang ${ }^{4}$, Tzu-Hsueh Tsai ${ }^{5}$ and DingKwo $\mathbf{W u}^{5^{*}}$

1 Department of Allied Health Care, Division of Medical Imaging, Ditmanson Medical Foundation, Chia-Yi Christian Hospital, Taiwan

2 Department of Medicine, Division of Gastroenterology and Hepatology, Ditmanson Medical Foundation, Chia-Yi Christian Hospital, Taiwan

3 Department of Surgery, Division of Urology, Ditmanson Medical Foundation, Chia-and Yi Christian Hospital, Taiwan

4 Department of Allied Health Care, Division of Pathology, Ditmanson Medical Foundation, Chia-Yi Christian Hospital, Taiwan

5 Department of Medical Imaging, Kaohsiung Medical University Hospital, Taiwan

\section{*Corresponding author: \\ Ding-Kwo Wu}

Department of Medical Imaging, Kaohsiung Medical University Hospital, Taiwan.

”dkwu@kmu.edu.tw

Tel: +88673121101

Fax: +88673212062

Citation: Lin ZC, Lin II, Chou CK, Shen CH, Wang WC, et al. (2018) Malignant Renal Epithelioid Angiomyolipoma with Liver Metastases Managed with Transarterial Chemoembolization (TACE): A Rare Case Report and Review. J Rare Disord Diagn Ther. Vol.4 No.4:19 
melanotic [human melanoma black-45 (HMB-45)] stain, on the other hand, allows differentiation with RCC. "Malignant" EAML is clinicially diagnosed when repeated local recurrence or remote metastases are identified [1]. When pathological diagnosis favors $A M L$ but without typical radiological configurations for CAML, clinician should be aware of EAML which may come across with high incidence of local recurrence and metastases, and thus, longterm monitoring with regular imaging surveilence is warranted.

\section{Case Report}

A 52-year-old female presented to a local hospital emergency room with sudden onset of left flank pain in July 2008. No kown underlying medical disease or family history of tuberous sclerosis could be traced. The laboratory tests showed only anemia (hemoglobin 9.5 grams per deciliter). No gross hematuria or occult blood was noted. Ultrasonography showed a calcified mass about $8 \mathrm{~cm}$ in diameter over left upper quadrant. Initial abdominal CT revealed a heterogenously enhanced mass, about $8.5 \mathrm{~cm}$ in diameter, at the upper pole of left kidney with spotty calcification, scattered necrosis, macroscopic fat spots at central portion and prominent vascularities at peripheral region (Figure 1). Associated imaging feature including perirenal hemorrhage was identified. No enlarged lymph nodes, intravascular tumor thrombi or hydronephrosis could be noticed. Preliminary radiological diagnosis including $\mathrm{RCC}$ and $\mathrm{AML}$ were made. She then searched for second opinion, asked to transfer to medical center and underwent left radical nephrectomy about 10 days later. Pathological diagnosis consistent with AML was made at the medical center, with epithelioid cells which are positive for HMB-45 and SMA, and negative for C-KIT (CD117) and cytokeratin, which are typical markers for RCC. Two years after initial diagnosis, abdominal CT showed local recurrence and retroperitoneal metastases abutting left iliopsoas muscle. In between 2011 and 2016, repeated tumor exicision, partial gastrectomy, partial colectomy of descending colon and splenectomy, were performed for recurrent tumor as well as for adjacent locally-invaded organs. Besides surgery, thalidomide $50 \mathrm{mg}$ per day had ever been tried for tumor control but ceased one week later due to side effects of intolerable fatigue in 2012. Transarterial embolization (TAE) was perfomed once with gelfoam pledgets via left inferior phrenic and left superior gluteal arteries for recurrent tumor at left subphrenic and iliopsoas muscle regions. Radiation therapy with total dosage of 4500 centigray (cGy) and 6000cGy, respectively, for left pelvic tumors were also conducted. Followe-up imaging study in 7 months reveals fatty degeneration within the bulky tumor contour but without gross shrinkage in size (Figure 2). Multiple liver metastases were noticed about 8 years from initial presentation and the laregest one sized about $5.8 \mathrm{~cm}$ in diameter. Pathological investigation comfirmed the diagnosis of metastatic hepatic tumors from AML (immunohistochemical stains : positive for HMB-45, Melan-A; and negative for cytokeratin and leukocyte common antigen, which are positive for RCC tumor) (Figure 3). The possibility of malignant EAML had also been mentioned in terms of marked cellular atypia, apparent necrosis and prior history of tumor recurrence and metastasis. Through reviewing of the medical literature, no relevant information regarding TACE treatment of liver metastases from EAML had been documented. Thus, we conducted twice TACE for the liver metastases (Figure 4) with anticancer regimen of $20 \mathrm{mg}$ of epirubicin mixed with $10 \mathrm{ml}$ of Lipioidol. The Lipiodol deposits didn't reach all areas of the tumor equally and that some areas of the tumor lacked Lipioidol. Unfortunately, the treatments failed along with rapid progression of non Lipiodol-targeted tumors in bilateral hepatic lobes (Figure 5) and recurrent tumor involving the stomach resulted in gastric bleeding. About 3 months later, she died of disease progression and septic shock related to intra-abdmoinal infection in 2017. Total survival time is approximately 9 years from the initial diagnosis.

\section{Discussion}

EAML is a rare variant of the AML with mean patient age of 40 years old and with even gender distribution, while CAML is predominant in the female with mean age of about 50 years old [2]. Typical AML is easy to make radiological diagnosis when presented with abundant macroscopic fat. Upon reviewing articles, imaging studies of renal EAML display minimal or no fat component and constitute more features of aggressive tumor characteristics $[4,5]$. Our case depicted typical characteristics of

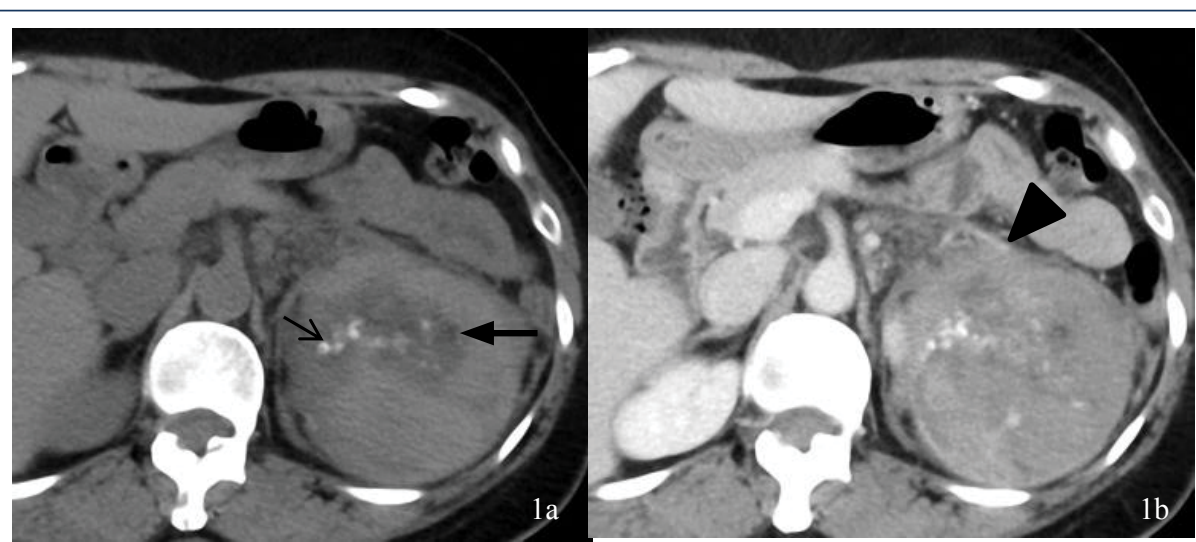

Figure 1 Initial CT evaluation with pre- and post-contrast abdominal CT images. A) Pre-contrast axial abdominal CT images showed a hyperattenuating mass lesion with spotty calcification (thin arrow), scattered necrosis and macroscopic fat spots at central portion (CT attenuation value of -15 Hounsfield units, thick arrow). B) Post-contrast axial abdominal CT images revealed heterogenous enhancement with prominent vascularities (arrowhead) at peripheral region. 
EAML in terms of bulky size, renal sinus fat invasion, heterogenous enhancement, hyperattenuation on non-contrast $\mathrm{CT}$, tumor necrosis, hemorrhage and locally aggressive behavior. However, small EAML presented with homogenous enhancement had also been reported $[3,5]$. In our case, no adjacent lymphadenopathy, no intratumoral aneurysm, no tumor invasion to renal vein or inferior vena cava, nor was metastasis identified; those of which were more consistent with RCC. The literature review indicated that intratumoral calcification more commonly found in RCC, was not a diagnostic criterion that could differentiate between RCC and EAML [3-5]. We don't know exactly why the occurrence of local lymph node involvement is less commonly encountered in EAML. But, as seen in our patient with longer follow-up up to 8 years, and also according to Froemming et al. report presented, local lymph node involvement in EAML may be unusual [5]. Small foci of fatty component identified in the tumor raise the possibility in favor of EAML [3]. When it comes to aggressive tumor characteristics, EAML and RCC are hardly differentiated radiologically. With the aid of immunohistochemical stain, PEComa shows reactivity on myogenic and melanocytic markers, such as HMB-45, SMA, Melan-A, which exclude a diagnosis of RCC. Some clues in favor of malignant EAML include large tumor size, eminent necrosis, frequent mitosis, atypical cell counts, and Ki-67 percentage score [5-9]. However, pathological predictors of the malignant EAML are still under debate. Clinically, the diagnosis of malignant EAML is made once distant metastases occurred.
The treatment of EAML remains controversial and no effective therapy other than surgery. Malingnat EAML with remote metastases usually has poor prognosis and surgery may not be optimised for multiple metastases. Adjuvant chemotherapy with doxorubin or anti-sarcoma regimen has variable efficacy on clinical response. Metastatic PEComa shows mTORC1 activation and genetic evidence of alteration in tuberous sclerosis complex (TSC1/TSC2) repressor. mTOR inhibitor had been used in some selective cases of malignant EAML with disease-free outcome, but in some other cases, progressive disease and fatal outcome had issued [9-12]. As there is no previous literature report addressing the optimized treatment protocol of TACE for the management of liver metastases from EAML. Thus, in recognition of multiple liver metastases, we had conducted TACE twice within 14 weeks intervals. We used a 4 French (Fr.) Yashiro catheter (Terumo, Japan) to engage the celiac trunk and co-axially place a 2.7 Fr. Progreat microcatheter (Terumo, Japan) placed in the right hepatic artery to deliver mixture of Lipioidol, contrast medium and epirubicin, and, followed by embolization of the feeder arteries with Gelfoam (Upjohn, USA) pledgets. As a treatment response, some tumors presented with compact lipioidol retention showed remarkable size reduction, but on the other hand, those with little or no Lipiodol deposition showed disease progression. Although based on the Modified Response of Evaluation Criteria in Solid Tumors (mRECIST) criteria, the overall treatment outcome was graded as disease progression. However, there were quite a few distictive

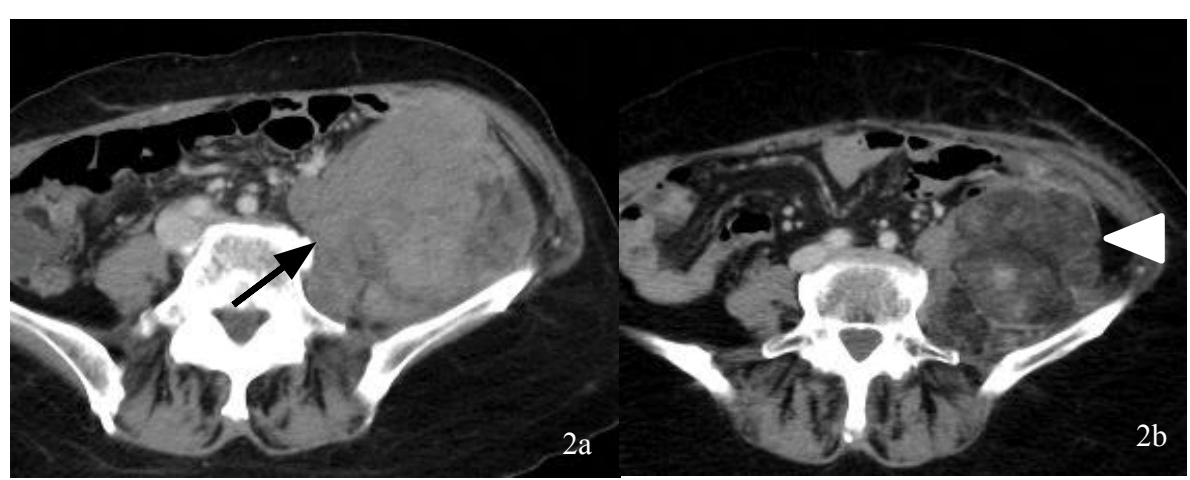

Figure 2 Comparative CT images to evaluate radiotherapy response. A) Pre-radiotherapy pelvic CT images revealed bulky recurrent/metastatic left pelvic tumor mass. (thick arrow). B) Post-radiotherapy images [Intensity modulated radiation therapy (IMRT), 6000cGy and 4000cGy, respectively, for left pelvic tumors) showed effective local tumor control for about 2 years, in terms of fatty degeneration and shrinkage of tumor dimension. (white arrowhead).

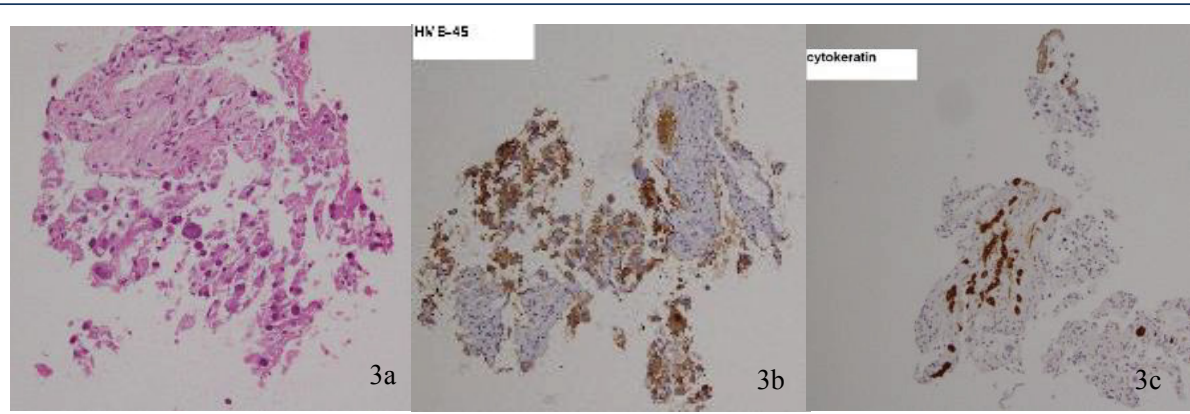

Figure 3 Hematoxylin and eosin stain (H\&E) and immunohisochmical stain of liver needle biopsy specimen. A) Showed epithelioid tumor cells with eosinophilic cytoplasm, centrally located nuclei with hyperchromatism, pleomorphism and marked tumor necrosis. B) Showed positive HMB-45 stain. C) Shows negative cytokeratin stain, respectively. 
nodules graded as complete remission and partial remissions (Figure 5C and 5D) in specific zone of the liver. In conjunction with moderately hypervascular nodules of varying size scattered in the right hepatic lobe on digital subtraction angiography (DSA), moderate amount of Lipiodol, i.e., $10 \mathrm{ml}$ mixed with $80 \mathrm{mg}$ of Epirubicin, might have yielded considerably better respone than anticipated. In retrospect, based on our preliminary experience dealing with few TACEs of angiosarcoma of the liver, it might be considered that our TACE formula in this case be adjusted, with increase of epirubicin dosage up to more than $80 \mathrm{mg}$, aimed preferentially for tumor toxicity rather than for predominating embolization. In the end, we might just have done partial TACE with low-dose of epirubicin mixed with Lipiodol and achieved tumor progression status on post-TACE follow-up protocol evaluated with mRECIST criteria for solid tumors. Indeed, there were sustantial declines of tumor vascularities appreciated on the $2^{\text {nd }}$ pre-TACE hepatic arteriography taken in 2 months (Figure 5C and 5D). The pathogenesis of renal EAML remains unclarified and demostrates malignant potential in light of local recurrence and distant metastasis. Initial presentation of aggressive tumor behavior with local recurrence or metastasis occur in about half of cases and $30 \%$ of them died of disease [8]. When remote metastases occured mainly in the lung and liver, relatively poor outcome had been documented. In the current case, TACE alone may not be appropriate in terms of bulky initial tumor burden and rapid tumor progression. Further investigation of optimal mode of therapy for malignant EAML is required.

\section{Conflict of Interest}

Authors have declared that there is no conflict of interest.

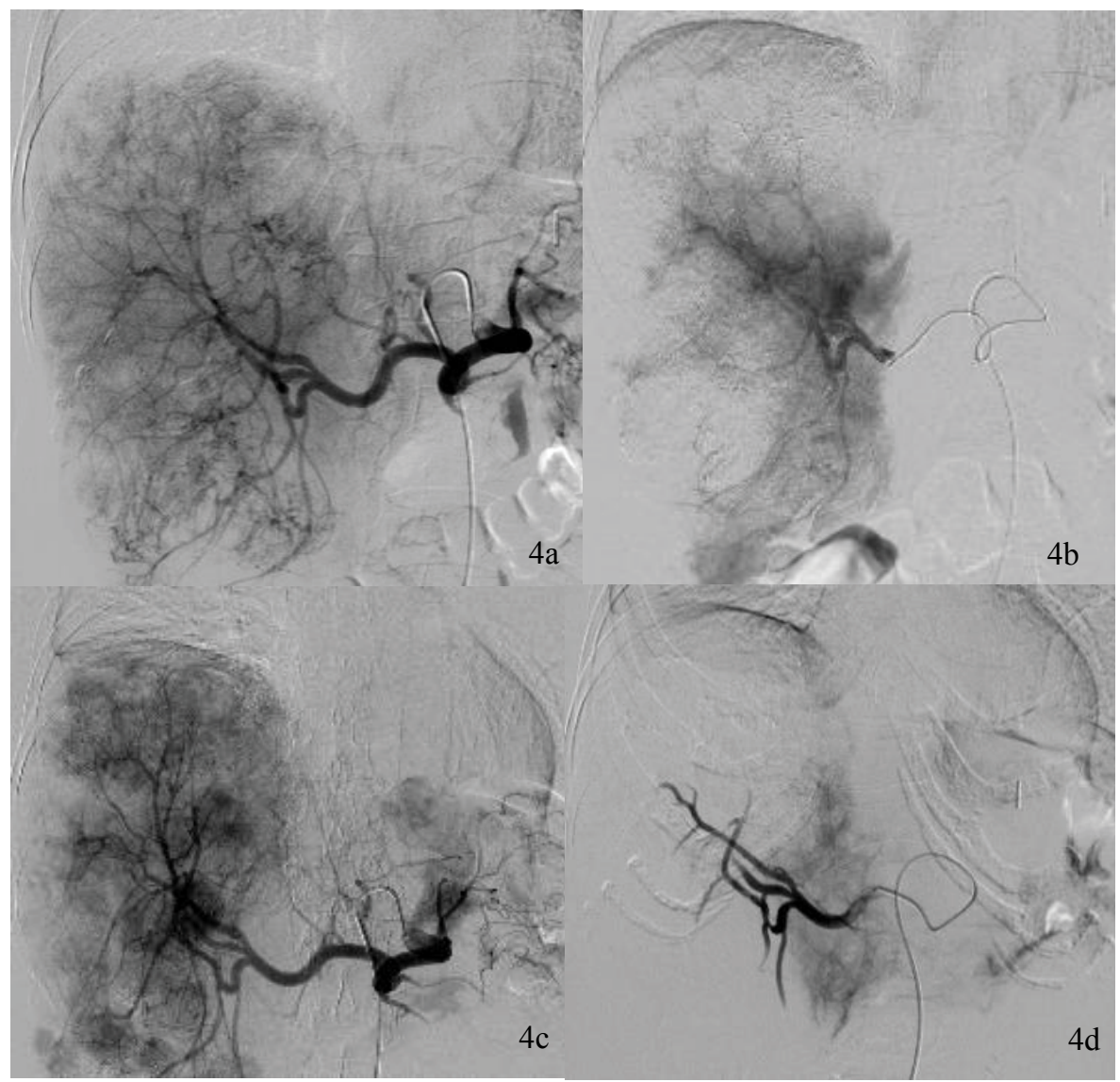

Figure 4 1st and 2nd pre-TACE and post-TACE digital subtraction hepatic arteriographies. A) 1st pre-TACE celiac arteriography showed multiple tumors with moderately hypervascular stain in the right hepatic lobe. B) Post 1st TACE right hepatic arteriography documented successful devascularization of feeder arteries. C) After 14 weeks from 1st TACE, 2nd celiac arteriography depicted sustantial declines of tumor vascularities and shrinkage of tumor burden in the right hepatic lobe as compared with 4A. D) Post-2nd TACE right hepatic arteriography documented high-grade devascularization of feeder arteries. 


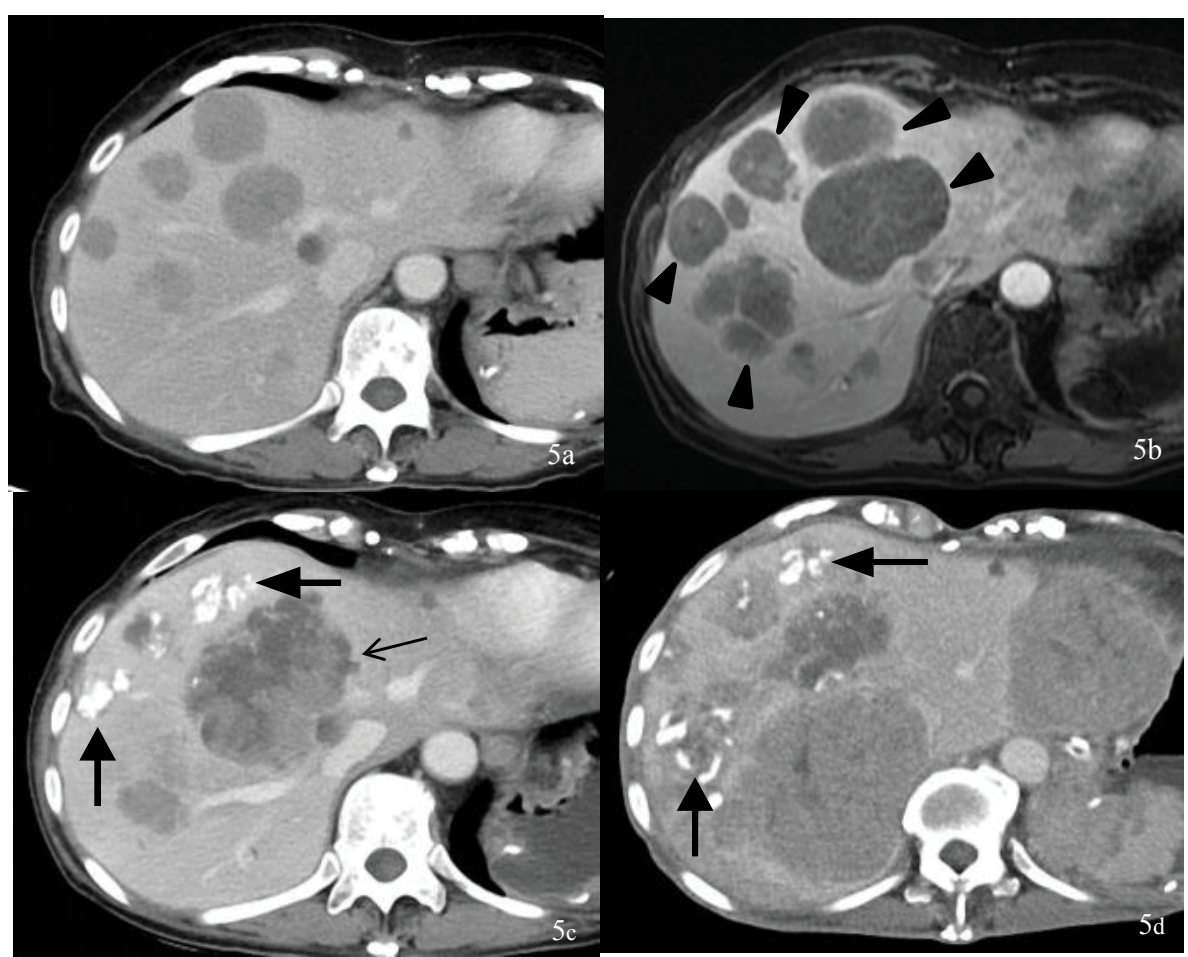

Figure 5 Serial follow-up images of liver metastases. A) One month prior to 1st TACE, enhanced CT showed multiple hypo attenuating liver metastases of varying size predominating in right hepatic lobe. B) T1 weighted enhanced magnetic resonance image (MRI), done on the same day of TACE, depicted significant interval worsening (arrowheads) as compared with 5A. C) 2 months post 1st TACE follow-up CT image. D) 2 months post 2nd TACE follow-up CT image. In $5 \mathrm{C}$ and 5D images, some tumors with compact Lipioidol retention (white spots, thick arrows) showed promising size reduction, but, in the others (thin arrows) with little or no Lipiodol deposition showed significant disease progression.

\section{References}

1. Guo B, Song H, Yue J, Li G (2016) Malignant renal epithelioid angiomyolipoma: A case report and review of literature. Oncol Lett 11: 95-98.

2. Vicens RA, Jensen $C T$, Korivi BR, Bhosale PR (2014) Malignant renal epithelioid angiomyolipoma with liver metastases after resection: a case report with multimodality imaging and review of the literature. J Comput Assist Tomogr 38: 574-77.

3. Schieda N, Kielar AZ, Al Dandan O, McInnes MDF, Flood TA, et al. (2015) Ten uncommon and usual variants of renal angiomyolipoma (AML): radiologic-pathological correlation. Clin Radiol 70: 206-220.

4. Tsai CC, Wu WJ, Li CC, Wang CJ, Huang CH, et al. (2009) Epithelioid angiomyolipoma of the kidney mimicking renal cell carcinoma: a clinicopathologic analysis of cases and literature review. Kaohsiung J Med Sci 25: 133-140.

5. Froemming AT, Boland J, Cheville J, Takahashi N, Kawashima A, et al. (2013) Renal epithelioid angiomyolipoma: imaging characteristics in nine cases with radiologic-pathologic correlation and review of the literature. AJR Am J Roentgenol 200: W178-W186.

6. Brimo F, Robinson B, Guo C, Zhou M, Latour M, et al. (2010) Renal epithelioid angiomyolipoma with atypia: a series of 40 cases with emphasis on clinicopathologic prognostic indicators of malignancy. Am J Surg Pathol 34: 715-722.

7. Li J, Zhu M, Wang YL (2012) Malignant epithelioid angiomyolipoma of the kidney with pulmonary metastases and p53 gene mutation. World J Surg Oncol 10: 213.

8. Luo J, Liu B, Wang Y, Li J, Wang P, et al. (2014) Comprehensive clinical and pathological analysis of aggressive renal epithelioid angiomyolipoma: report of three cases. OncoTargets Ther 7: 823-827.

9. Lei JH, Liu LR, Wei Q, Song TR, Yang L, et al. (2015) A Four-Year Follow-up Study of Renal Epithelioid Angiomyolipoma: A MultiCenter Experience and Literature Review. Sci Rep 5: 10030.

10. Wagner AJ, Malinowska-Kolodziej I, Morgan JA, Qin W, Fletcher CDM, et al. (2010) Clinical activity of mTOR inhibition with sirolimus in malignant perivascular epithelioid cell tumors: targeting the pathogenic activation of mTORC1 in tumors. J Clin Oncol 28: 835-840.

11. Gennatas C, Michalaki V, Kairi PV, Kondi-Paphiti A, Voros D, et al. (2012) Successful treatment with the mTOR inhibitor everolimus in a patient with perivascular epithelioid cell tumor. World J Surg Oncol 10: 181.

12. Schwartz LH, Litiere S, Vries DV, Ford R, Gwyther S, et al. (2017) RECIST 1.1 - Update and Clarification: From the RECIST Committee. Eur J Cancer 62: 132-137. 